\title{
In Silico Binding Interactions Of Dehalogenase (Dehe) With Various Haloalkanoic Acids
}

Nur Illani Abd Halin ${ }^{1}$, Fahrul Huyop ${ }^{2}$, Tengku Haziyamin Tengku Abdul Hamid ${ }^{1}$, Khairul Bariyyah Abdul Halim ${ }^{1}$, Azzmer Azzar Abclul Hamid ${ }^{1 *}$

${ }^{1}$ Department of Biotechnology, Kulliyyah of Science, International Islamic University Malaysia, Jalan Sultan Ahmad Shah, Bandar Indera Mahkota, 25200, Kuantan, Pahang, Malaysia ${ }^{2}$ Department of Biotechnology and Medical Engineering, Faculty of Biosciences and Medical Engineering, Universiti Teknologi Malaysia, 81310, Johor Bahru, Johor, Malaysia *Corresponding author: azzmer@iium.edu.my

This is an open access article distributed under the Creative Commons Attribution License, which permits unrestricted use, distribution, and reproduction in any medium, provided the original work is properly cited

\section{ARTICLE DETAILS}

Article history:

Received 27 September 2016 Accepted 13 December 2016 Available online 10 January 2017

\section{Keywords:}

dehalogenase, DehE, Haloalkanoic acid, molecular docking simulation

\section{ABSTRACT}

Synthetic haloalkanoic acid (HA) is one of the synthetics compounds that can be found as active ingredients in herbicides. These compounds are known to pollute our agriculture land due to their toxicity, thus may cause serious environmental and health problems. Biological process such as microbial dehalogenation degrades the harmful compounds and prevents their migration into groundwater source. For instance, Rhizobial Dehalogenase E (DehE) could catalyze these HA compounds and convert them into hydroxylated compounds which are less harmful to the environment. In previous study, DehE was considered to degrade many HA compounds with different Km values. However, the binding interaction of this enzyme towards many HA substrates is still unclear. In this study, docking simulation has been performed to determine the affinity of active site residues of DehE towards 15 HA compounds. Tribromoacetic acid (TBA) was identified to be the most favourable substrate for DehE which has the lowest binding energy $(-6.48 \mathrm{Kcal} / \mathrm{mol})$ compared to other haloalkanoic acids. Size of halogen and hydrogen bond numbers are the contributing factor for dehalogenase affinity towards its substrates. Besides, it was found that Trp34, Phe37 and Ser188 served as binding residues and Phe37 was mostly interacted and bound with all of the tested HA compounds. This findings provides an opportunity for rational design of haloacid dehalogenase especially to DehE.

\section{INTRODUCTION}

Synthetic organohalogens are being synthesized as active compounds in the production of pesticides and herbicides. These compounds including haloalkanoic acids (HA) are widely dispersed in agriculture area and are toxic to the environment. Consequently, these HA compounds could contribute to human health problems and environmental pollution.

Malaysia is among other countries in Asia Pacific that use pesticides extensively in agriculture. About 1.5 million hectare of lands were used for rubber trees cultivation and 0.6 hectares are devoted for oil palm trees [1]. According to Awang and his co-workers, high number of pesticides used in agriculture was contributed to almost $50 \%$ total number of 5,152 cases of human poisoning in Malaysia. Further, there are certain pesticide compounds that contribute towards poisoning cases such as $2.6 \%$ of poisoning has caused by organochlorine compounds [2].

In bioremediation aspect, microbial dehalogenase enzyme has been discussed and proven to degrade variety of halogenated compounds [3, 4]. All dehalogenases were classified into three groups namely hydrolytic, haloalcohol and cofactor-dependant [5]. The primary interest of this study is the haloacid dehalogenase, or specifically haloacid dehalogenase E (DehE) from soil Rhizobium sp. RC1. Haloacid dehalogenase is grouped under hydrolytic dehalogenase which could hydrolyze haloalkanoic acids and converted into hydroxyl acid compound [6].

DehE from soil Rhizobium sp. RC1 could act on all isomers (D- and L-) of chlorinated and brominated compounds but showing no specificity towards any substrate [7]. Recently, the structure of DehE with 12 active site residues was modeled using DehI enzyme from Pseudomonas putida PP3 as a template [8]. Among the active site residues, only Phe37, Try34 and Ser188 were confirmed to be as binding residues for D- and L-2chloropropionic acid (D- and L-2CP) [9]. To date, these three binding residues were only been identified with very limited substrates (only Dand L-2CP). Furthermore, there is a lack of information regarding molecular interaction between DehE and HA substrates. Thus, to understand the binding interaction at molecular level, molecular docking technique has been employed to investigate the affinity of DehE towards substrates. This research would provide a foundation for mutagenesis studies to design haloacid dehalogenase for potential application in bioremediation.

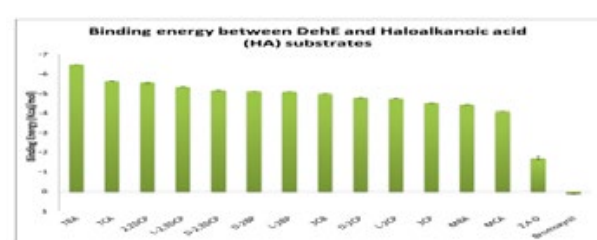

\section{EXPERIMENTAL}

2.1 Data Set for Molecular Docking

Molecular structure of DehE was modeled by SWISS-MODEL program through Expasy web-server as described earlier [8]. The 15 HA compounds were retrieved from PubChem project database [10]. All the ligand structures in PDB format (.pdb) were used as starting materials for molecular docking simulation.

2.2 Molecular Docking between DehE and HA compounds

In this study, docking simulations between receptor (DehE) and substrates (HA) were performed using AutoDock Tool software (ADT) version 4.2.6 [11]. In the initial steps of the docking simulation, polar hydrogen atom, partial charges, Kollman charges, Gasteiger charges were added for molecular preparation. Subsequently, molecular coordinates in PDBQT file were created including atomic partial charges and atom type's information. Grid map type was set up in the region of active site $(46 \times 52 \times 54)$ and spacing of $0.375 \AA$ using AutoGrid. Thus, the location of docking was specified to the active site of DehE (Trp34, Ala36, Phe37, Asn114, Tyr117, Ala187, Ser188, Asp189, Tyr265, Phe268, Ile269 and Ile271). Then, Lamarkian genetic algorithm (LGA) was employed for 100 docking protein-substrates runs. After docking simulation, the results of docked conformations were written in a $\log$ file (.dlg). All the information on clustering and interaction energies was reported in the form of histogram. The highest conformation cluster with the lowest binding energy was chosen for structural analysis.

2.3 Structure Visualization of DehE Complexes

The result from docking was visualized by using PyMol software for high quality of molecular graphics image [12]. Besides viewing, calculation of hydrogen bond distance was also performed using this software. Apart from that, the schematic 2-D representation for substrate binding was generated and viewed using LigPlot software [13].

\section{RESULTS AND DISCUSSION}

3.1 Binding Energies of DehE and haloalkanoic acids (HA) complex Based on the binding energies obtained from molecular docking simulations (Figure 1), the most favorable compound for DehE enzyme is Tri-bromoacetic acid (TBA) which has the lowest binding energy $(-6.48 \pm 0.00 \mathrm{kcal} / \mathrm{mol})$. Meanwhile, Bromoxynil compound becomes less preferable substrate for DehE when the docking analysis resulted to the highest binding energy level $(+0.12 \pm 0.01 \mathrm{kcal} / \mathrm{mol})$. These energy values indicate that DehE has stronger binding affinity for TBA and low preference towards Bromoxynil. Moreover, the result also showed decreasing affinity from tri-halogenated to mono-halogenated acids. It is contradicted with the previous study, which describe a decreasing $\mathrm{Km}$ value for chloro and bromoacetate from monohalogenated to tri-halogenated acetate [7]. According to Commandeur and his co-workers, the presence of more halogen carbons in one molecule 
causes difficulty for the enzyme to facilitate substrate degradation [14]. However, DehE affinity shown in this study is proportional to the number of halogen in the substrate.

High energy level obtained for DehE-Bromoxynil complex might due to the size of Bromoxynil substrate which led to destabilization of interaction in the active site of DehE. The structure of Bromoxynil is categorized as a bulky aromatic compound with carbon-ring attached to two bromine atoms. The type of HA substrate has profound effect on the vulnerability of a compound to be dehalogenated. According to Allpress and Gowland (1998), aromatic compound is complicated to be metabolized due to the cleavage of the ring structure and it should be performed after removal of the halogen compound. Besides Bromoxynil, 2, 4-D is also classified under the same category and it was shown that the DehE enzyme had very low affinity towards this compound (Figure 1).

Docking result also revealed that each of brominated compounds has lower binding energy than chlorinated compounds. Table 1 shows the comparison for binding energy between two different types of halogen compounds from mono- to tri-halogenated. Previously, Huyop and his colleagues have studied the $\mathrm{Km}$ value for Brominated substrates and they found that the values were lower compared to chlorinated substrates [7]. Besides, Allison et al. (1983) explained the brominated compound was the most preferable substrate because the time for its degradation was faster than chlorinated compound. According to Bronowska (2011), the increasing size of halogen atom was a contributing factor to the strong hydrogen bonds formation and high affinity of enzyme towards substrate. It is clear in our study that the size of halogen atom has a significant effect on the binding affinity of dehalogenase $(\mathrm{Br}>\mathrm{Cl})$.

\begin{tabular}{ll} 
Table 1 Comparison of binding energy between two different halogens \\
\begin{tabular}{ll}
\hline Mono-halogenated & Binding Energy \\
compound & Kcal/moD
\end{tabular} \\
\hline Monobromonated (MBA) & -4.43 \\
Monochloronated (MCA) & -4.09 \\
\hline Tribalogenated compound & $\begin{array}{l}\text { Binding Energy } \\
\text { (Kcal/moD }\end{array}$ \\
\hline $\begin{array}{l}\text { Tribromonated (TBA) } \\
\text { Trishloromated (TCA) }\end{array}$ & -6.48 \\
\hline
\end{tabular}

3.2 Intermolecular Contacts in Substrate binding

In intermolecular analysis, several amino acids were confirmed to interact with each substrate in the active site through hydrogen bond formations. As seen in many complexes, hydrogen bonds were formed between carboxyl groups $(\mathrm{COOH})$ of $\mathrm{HA}$ and hydrogen $(\mathrm{H})$ atom of amino acid within the distance of $\sim 1.6 \AA$ to $\sim 2.5 \AA$. This value is corroborated with the measurable distance of hydrogen bond which can typically be built in $3.0 \AA$ [15]. Referring to Figure 2, all hydrogen bonds were mostly formed by Phe37, Trp34 and Ser188 residues. However, for DehE-Bromoxynil, only a single hydrogen bond was formed between Phe37 residue and the substrate. We also found that the distances between Trp34 and all HAs are in shorter range compared to other binding residues (Phe 37 and Ser188). Thus, our result suggests that tryptophan is a suitable amino acid for substrate recognition and play important role in binding affinity. This is because Tryptophan is a non-polar group of amino acid and it could also interact with substrate through van der waals interactions [15].

The hydrogen bonding between DehE and HA was supported by hydrophobic contacts as shown in Figure 3. For DehE-TBA complex two hydrophobic amino acids came into contact with TBA while the rest involved in the formation of hydrogen bonds. In contrast, Bromoxynil has many hydrophobic contacts surrounding its hydrogen bond residues although only limited hydrogen bonds detected to hold Bromoxynil in the active site. Therefore, the weak interaction between DehE and Bromoxynil complex might be due to the low hydrogen bond number

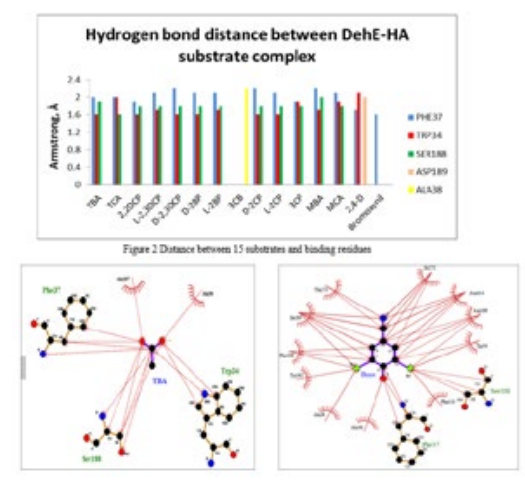

Figure 3 Close hydrophobic contacts in DehE active site. (a) Ala187 and Ala36 residues that involved in the contacts for TBA substrae. (b) Hydrophobic contact of DehE-Bromoxynil complex. There are 13 residues involved in the hydrophobic interaction

In our previous study, it was demonstrated that DehE has twelve conserved active site residues namely Trp34, Ala36, Phe37, Asn114, Tyr117, Ala187, Ser188, Asp189, Tyr265, Phe268, Ile269, and Ile271[8]. However, only a few residues would facilitate substrate recognition. Our previous study suggest that different substrates will result in varies binding residues. However most substrates formed hydrogen bond with Trp34, Phe37 or Ser188. Interestingly, Phe37 was determined to form hydrogen bond with all tested haloalkanoic acids except for 3CB (Figure 2). This highlighted the role and function of Phe37 as important binding residue for DehE. In contrast, 3CB was hydrogen bonded with irregular binding residue which is Asp189. This amino acid along Phe37 and Trp34 have been bound with 2,4-D through hydrogen bond interactions. In previous study, Asp 189 was also predicted as catalytic residue which could activate water molecule for direct attack mechanism [16].

\section{CONCLUSION}

In this study, docking simulations were used to determine the binding affinity for DehE towards many HA substrates. The lowest binding energy predicted for TBA suggests that TBA is the most preferable substrate. We also found that the size of halogen and hydrogen bonds number may play an important role on substrate recognition and enzyme affinity. Analysis of enzyme-substrates interactions from the docking complexes revealed that the three binding residues which are Trp34, Phe37 and Ser188, crucial for substrate attachment. Among them, Phe37 is the most important residue due to its interaction to almost all tested substrates.

Acknowledgement

We are grateful to IIUM and Ministry of Higher Education for supporting this project through FRGS grant (FRGS15-208-0449).

\section{References}

[1] Awang, R., A. Latiff, M. Majid, and A. Dzulkifli. 2011. Case study: Malaysian information service on pesticide toxicity. Encyclopaedia of occupational health and safety. Geneva, Switzerland: International Labour Organization. 22.

[2] Chong, E., H. S. Tara, M. Jusoh, and K. Tham. 1989. Toxicological analytical facilities in Malaysia.

[3] Copley, S. D. 1998. Microbial dehalogenases: enzymes recruited to convert xenobiotic substrates. Current opinion in chemical biology. 2(5): 613-617.

[4] Kurihara, T., N. Esaki, and K. Soda. 2000. Bacterial 2-haloacid dehalogenases: structures and reaction mechanisms. Journal of Molecular Catalysis B: Enzymatic. 10(1): 57-65.

[5] Slater, J. H., A. T. Bull, and D. J. Hardman. 1995. Microbial dehalogenation. Biodegradation. 6(3): 181-189.

[6] Field, J., and R. Sierra-Alvarez. 2004. Biodegradability of chlorinated solvents and related chlorinated aliphatic compounds. Reviews in environmental Science and Bio/technology. 3(3): 185-254.

[7] Huyop, F. Z., T. Y. Yusn, M. Ismail, R. A. Wahab, and R. A. Cooper. 2004. Overexpression and characterisation of non-stereospecific haloacid Dehalogenase E (DehE) of Rhizobium sp. Asia Pac. J. Mol. Biol. Biotechnol. 12: $15-20$.

[8] Hamid, A. A. A., E. L. Wong, K. H. Joyce-Tan, M. S. Shamsir, T. H. T. A. Hamid, and F. Huyop. 2013. Molecular modelling and functional studies of the nonstereospecific $\alpha$-Haloalkanoic acid Dehalogenase (DehE) from Rhizobium sp. RC1 and its association with 3-chloropropionic acid ( $\beta$-chlorinated aliphatic acid). Biotechnol Biotechnol Equip. 27: 3725-3736.

[9] Hamid, A. A. A., T. H. T. A. Hamid, R. A. Wahab, and F. Huyop. 2013. Identification of functional residues essential for dehalogenation by the non-stereospecific $\alpha$-haloalkanoic acid dehalogenase from Rhizobium sp. RC1. Journal of Basic Microbiology: Accepted for publication.

[10] Wang, Y., J. Xiao, T. O. Suzek, J. Zhang, J. Wang, and S. H. Bryant. 2009. PubChem: a public information system for analyzing bioactivities of small molecules. Nucleic acids research. 37(suppl 2): W623-W633.

[11] Goodsell, D. S. 2009. Computational docking of biomolecular complexes with AutoDock. Cold Spring Harbor Protocols. 2009(5): pdb. prot5200.

[12] DeLano, W. (2004). Use of PyMOL as a communications tool for molecular science. Paper presented at the ABSTRACTS OF PAPERS OF THE AMERICAN CHEMICAL SOCIETY.

[13] Wallace, A. C., R. A. Laskowski, and J. M. Thornton. 1995. LIGPLOT: a program to generate schematic diagrams of protein-ligand interactions. Cite this article as: Nur Illani Abd Halin, Fahrul Huyop, Tengku Haziyamin Tengku Abdul Hamid, Khairul Bariyyah Abdul Halim, Azzmer Azzar Abdul Hamid. In Silico Binding Interactions Of Dehalogenase (Dehe) With Various Haloalkanoic Acids. G. War. Sains1(1) (2017): 04-06 
Protein engineering. 8(2): 127-134.

[14] Commandeur, L. C., and J. R. Parsons. (1994). Biodegradation of halogenated aromatic compounds Biochemistry of microbial degradation (pp. 423-458): Springer.

[15] Petsko, G. A., and D. Ringe. 2004. Protein structure and function: Sinauer Associates Inc.

[16] Schmidberger, J. W., J. A. Wilce, A. J. Weightman, J. C. Whisstock, and M. C. Wilce. 2008. The Crystal structure of dehI reveals a new $\alpha$-haloacid dehalogenase fold and active-site mechanism. Journal of molecular biology. 378(1): 284-294.

fish product prepared with different salt concentrations. Int. Journal of Food Microbiology.73.61-70.

[21] Kaktcham P.M., Zambou N.F., Tchouanguep F.M., El-Soda and M. Choudary M.I. 2011. Antimicrobial and Safety properties of lactobacilli isolated from two Cameroonian traditional fermented foods. Open Access article Scientia Pharmaceutica.80 (1): 189-203. 